\title{
The Value of Endoscopic Ultrasonography in the Endoscopic Resection of Gastrointestinal Stromal Tumors
}

\author{
Jian-Wei $M i^{1}$ \\ Jia-Qi Wang ${ }^{2}$ \\ Jie $\mathrm{Liu}^{3}$ \\ Li-Xian Zhang' \\ Hong-Wei Du' \\ Dong-Qiang Zhao'
}

'Department of Gastroenterology, The Second Hospital of Hebei Medical

University, Shijiazhuang, 050000, People's

Republic of China; ${ }^{2}$ Basic Medical

College, Hebei Medical University,

Shijiazhuang, 050000, People's Republic of

China; ${ }^{3}$ Department of Trauma

Emergency, Medical Noncommissioned

Officer School, Army Medical University,

Shijiazhuang, 050000, People's Republic of

China
Correspondence: Dong-Qiang Zhao Department of Gastroenterology, The Second Hospital of Hebei Medical

University, No. 215 of West Peace Road,

Xinhua District, Shijiazhuang, 050000,

People's Republic of China

Tel +86031 I 66636179

Email drzhaodq_II24@I63.com
Objective: This study aimed to explore the clinical value of endoscopic ultrasonography (EUS) in the endoscopic resection of gastrointestinal stromal tumors (GISTs).

Methods: A retrospective study of 92 patients who were confirmed to have GISTs by endoscopic resection after EUS examination was conducted. The preoperative features of the EUS examination, ultrasound diagnosis, endoscopic resection methods, surgical procedures, complications, and complete degree of lesion resection were recorded. And 16 patients who were diagnosed by endoscopy and EUS and confirmed by surgical operation were included and analyzed in the subsequent part of the investigation (gastroscopy and EUS image analysis, EUS image and risk classification).

Results: The preoperative diagnosis rate of EUS and postoperative pathological diagnosis of GISTs was $78.7 \%(85 / 108)$, and the presence of a non-homogeneous echo and liquid anechoic zone in GISTs often indicated higher risk $(P<0.05)$. There was a positive correlation between tumor size and risk $(P<0.05)$.

Conclusion: The endoscopic resection of GISTs is feasible and safe. EUS is of great significance for the diagnosis and risk assessment of GISTs and can assist in the endoscopic resection of GISTs.

Keywords: endoscopic ultrasonography, endoscopic resection, gastric stromal tumor, risk assessment, complications

\section{Introduction}

Gastrointestinal stromal tumors (GISTs) are non-epithelial tumors of the digestive tract with varying degrees of malignant potential. ${ }^{1,2}$ Previous studies have shown that GISTs can occur throughout the gastrointestinal tract, but the majority of cases occur in the gastric body $(55.6 \%)$, small intestine $(31.8 \%)$, colorectum $(6.0 \%)$, and other parts of the gastrointestinal tract $(6.6 \%) .^{2}$ The main clinical symptoms of GISTs are gastrointestinal bleeding, abdominal pain, and mass compression. In recent years, with the popularization of endoscopy, it has been possible to detect small asymptomatic GISTs (diameter $<2 \mathrm{~cm}$ ) and small GISTs (diameter $<1 \mathrm{~cm}$ ). Among these tumors with high mitotic figures are often associated with high invasive biological behavior. ${ }^{3,4}$

Endoscopic ultrasonography (EUS) provides a basis for the early diagnosis of GISTs by identifying the characteristics of a tumor, while endoscopic resection, due to its advantages of minimal surgical trauma and rapid recovery, is an option for the early treatment of GISTs. Although the endoscopic and surgical treatment of GISTs 
remains controversial, recent prospective comparative studies and clinical studies with large sample sizes have confirmed the efficacy and safety of endoscopy in the treatment of GISTs. ${ }^{23,24}$ An EUS artificial intelligence system for GISTs is also being developed. ${ }^{25}$ The objective of the present study was therefore to explore the clinical value of EUS in the endoscopic resection of GISTs.

\section{Materials and Methods}

\section{Clinical Data}

\section{Subjects}

This study retrospectively analyzed the data of 92 patients with GISTs who underwent an endoscopic resection after receiving an EUS diagnosis at the Second Hospital of Hebei Medical University's Department of Gastroenterology between May 2012 and October 2018 and confirmation of the diagnosis by pathology and immunohistochemistry.

The study was conducted in accordance with the Declaration of Helsinki and was approved by the Ethics Committee of the Second Hospital of Hebei Medical University. The operation method and precautions were explained to the patients before the procedure, and all patients provided written informed consent for the endoscopic examination and endoscopic treatment as well as for the use of their data for the purposes of research. All endoscopic evaluations and operations were performed by two experienced chief physicians.

\section{Inclusion and Exclusion Criteria}

Inclusion criteria: (1) patients with a confirmed diagnosis of GISTs regardless of tumor diameter and/or mitotic index; (2) an endoscopic resection was performed; (3) patients with complete postoperative pathological and immunohistochemical data; (4) patients over the age of 18.

Exclusion criteria: (1) patients with severe heart, liver, kidney, and other organ complications or dysfunction; (2) patients with coagulation complications and dysfunction; (3) patients who had received other surgical treatment.

\section{Preoperative Preparation}

\section{Preoperative Routine Examination}

A chest X-ray, abdominal computed tomography, electrocardiogram, routine blood test, biochemical test, routine coagulation test, and four preoperative items were performed.

\section{Equipment}

The following equipment was used in this study: Fujinon EG-530 UT electronic ring-scanning ultrasound endoscope, Fujinon EG-530UR electronic sectorialscanning ultrasound endoscope, Fujinon EG-450CT5 miniature ultrasound probe endoscope, dual-knife (KD-655L/ Q), IT-knife (KD-612L), snare (X=30 mm, $Y=55 \mathrm{~mm}$, MTW), ligator (MBL-6-F, COOK), and hyaline cap (D-201-11804, Olympus).

\section{Surgical Procedure EUS}

After preparation, the gastric cavity was entered and rinsed clean, and the mucosal conditions of the tumor (including mucosal integrity, smoothness, presence of ulcers, and bleeding) were recorded for a preliminary evaluation of tumor size. EUS was then performed to record the origin level, size, echo characteristics (including type, uniformity, calcification, and liquid anechoic area), and internal blood flow of the tumor, as well as the presence of any lymph nodes adjacent to and surrounding the tumor.

\section{Endoscopic Resection}

The patient was examined in the standard gastroscopy position (left lateral position). An anesthesiologist performed intravenous anesthesia. According to the characteristics of the EUS images, the appropriate surgical methods were selected. The operation process, bleeding, and perforation was recorded.

\section{EBL}

Under the endoscope, the tumors were extracted into a hyaline cap using a ligator, the ligation ring was released, and the tumor was completely extracted into the ligation ring. The tumors were then removed from the root using a snare. After complete excision, the wound was observed for active bleeding and perforation before being closed with a purse-string suture using metal clips or metal clips combined with a nylon ring. The excised specimens were then confirmed to be complete and sent for pathological testing.

\section{ESD}

A hyaline cap was placed in the front end of the endoscope body. The edges of the lesion were marked using allophycocyanin, and a blue glycerol-fructose-methylene mixture was injected submucosally. A dual knife was used to incise the mucosa in a ring shape along the marker points. The dual knife and an IT knife were used to peel off the mucosa and submucosa layer by layer until the tumors were completely free. 


\section{ESE}

A hyaline cap was placed in the front end of the endoscope body. A dual knife was used to mark the tumors along the midline of the mucosa. A blue glycerol-fructosemethylene mixture was injected. The lifting sign was positive. A dual knife was then used to incise the mucosa along the marker points to expose the tumors, and the tumors were dissected using an IT knife.

\section{EFTR}

The EFTR procedure is the same as that of ESE except that the perforations are actively made when the tumor is dissected, meaning that the full thickness of the tumor is removed. After EFTR, the perforations were closed using titanium clips, titanium clips combined with a purse-string suture, or over-The-scope clips $\left(\mathrm{OTSCs}^{\mathbb{B}}\right)$. The specimens were then sent for analysis.

\section{STER}

A hyaline cap was placed in the front end of the endoscope body. A blue glycerol-fructose-methylene mixture was injected submucosally near the mass. The lifting sign was positive. A dual knife was used to remove the mucosa, separate the mucosa and submucosa, and build tunnels.

\section{Specimen Processing}

After the procedure, the specimens were immediately fixed in a $4 \%$ neutral formaldehyde fixative and sent for pathological testing and immunohistochemistry. The integrity of the lesion margin was determined.

\section{Statistical Methods}

Data were analyzed using statistical software SPSS 21.0. Normally distributed measurement data were expressed as mean \pm standard deviation $(x \pm \mathrm{SD})$, and non-normally distributed measurement data were expressed as the median \pm interquartile range ( $\mathrm{M} \pm \mathrm{QR})$. Count data were expressed as a percentage (\%). The normality of variables was tested using a W-test. Count data were evaluated using a $\chi^{2}$ test. Data with $T<5$ were evaluated using Fisher's exact probability method. $P<0.05$ was considered statistically significant.

\section{Results}

\section{General Data}

A total of 92 patients were included in the current study (43 male, 49 female). The ages of the patients ranged from 30 to 82 years, with an average age of $60.07 \pm 9.01$ years.

\section{Gastroscopy and EUS Image Analysis}

Under general endoscopy, 108 patients presented with hemispherical or spherical submucosal eminences. These were primarily located in the fundus of stomach $(57 / 108$, $52.7 \%)$, followed by the gastric body $(24 / 108,22.2 \%)$, gastric fundus junction $(15 / 108,14.0 \%)$, and cardia and subcardia $(8 / 108,7.4 \%)$, with the fewest occurrences in the gastric antrum $(4 / 108,3.7 \%)$. Congestion and erosion on the surface of the lesions were found in 12 patients (11.1\%), hyperemia and edema in seven patients (6.5\%), and apical depression in three patients (2.8\%).

Preoperative EUS revealed that 85 patients were suspected to have been diagnosed with GISTs. The main manifestations were hypoechoic, even or uneven, with or without internal liquid anechoic areas, and some with calcification or blood flow. Seven patients were suspected to have been diagnosed with leiomyoma and GISTs, three were suspected to have been diagnosed with leiomyoma, one was suspected to have been diagnosed with a schwannoma (and this case was the only one in the data center diagnosed as hyperechoic; this patient's brother was also suspected to have been diagnosed with high echogenic nerves as a young man). Twelve patients were only described with pathological characteristics.

A total of 85 patients were examined by circular EUS, 19 by a small probe, three by sectorial scanning, and one by a small probe combined with circular scanning. The EUS revealed that the majority of the GISTs originated from the muscularis propria $(104 / 108,96.3 \%)$, with the rest originating from the muscularis mucosae (4/108, $3.7 \%)$. A low echo was found in the lesions of 106 patients (98.2\%), an iso-echo in one patient $(0.9 \%)$, and a high echo in one patient $(0.9 \%)$; the echo was even in 70 patients and uneven in 38 patients. Scattered blood-flow signals within the lesions were found in seven patients (6.5\%), calcification in 10 patients $(9.3 \%)$, and anechoic fluid in 19 patients (17.6\%). See Figure 1.

\section{Endoscopic Treatment}

A total of 92 patients underwent endoscopic treatment, of which 89 (96.7\%) underwent complete pathological resection, two underwent ligation and had their partially excised specimens sent for pathological analysis, and one underwent elective surgery after consultation due to the deep location of the tumors and their abundant blood supply; the partially excised specimens from this patient were also sent for pathological analysis. Of these 92 patients, 37 


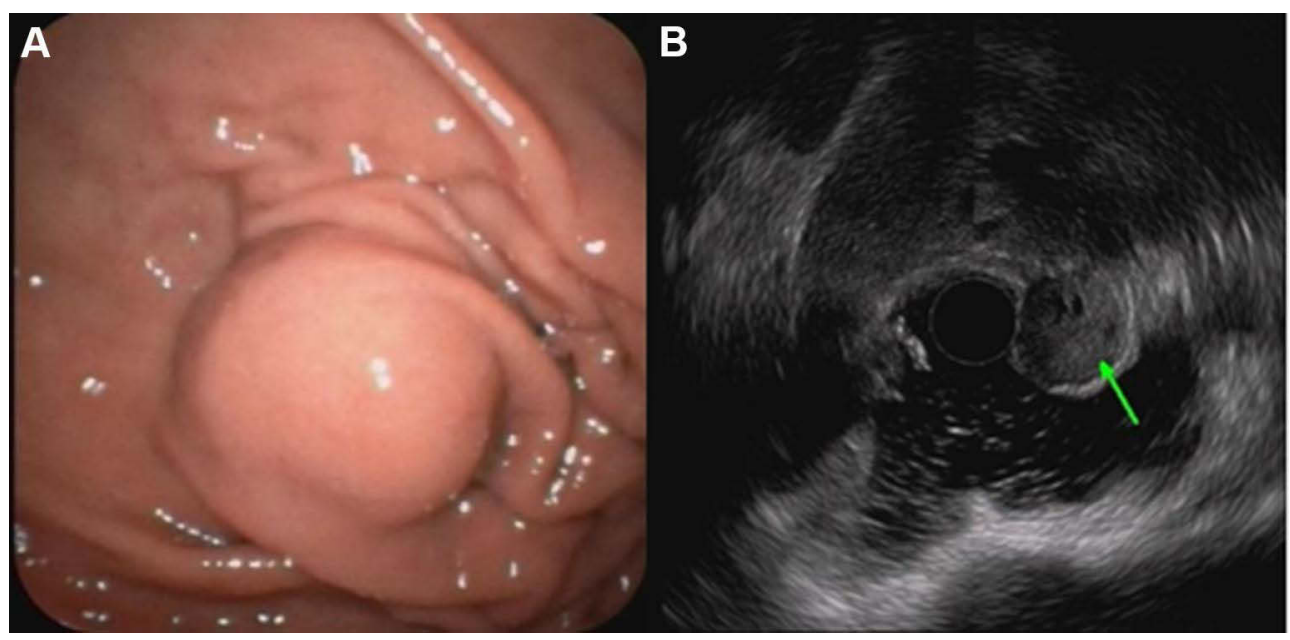

Figure I Endoscopic and endoscopic ultrasonography for gastrointestinal stromal tumor. (A) A submucosal tumor was detected in the gastric fundus by general gastroscopy. (B) Endoscopic ultrasonography showed that the lesion originated from the muscularis propria, was hypoechoic, and had a non-homogeneous echo and visible echo-free area; Doppler ultrasound did not identify blood flow; cross-section size: $2.0 \mathrm{~cm} * 2.5 \mathrm{~cm}$.

(40.2\%) underwent ESD, 27 (29.4\%) underwent ESE, 15 $(16.3 \%)$ underwent EFTR, nine $(9.8 \%)$ underwent EBL, and four $(4.3 \%)$ underwent STER.

Intraoperative bleeding occurred in two patients (2.2\%), and both of these cases occurred during ESE. Thermal biopsy forceps and titanium clamps were successfully used to stop the bleeding. Perforation occurred in 13 patients (14.1\%; cases of perforation during EFTR were not included), three of which occurred during EBL, six during ESD, and four during ESE. These perforations were successfully closed with titanium clips, titanium clips combined with a purse-string suture, or OTSCs ${ }^{\circledR}$. After the procedure, electrocoagulation syndrome occurred in seven patients (two cases after ESD, two after ESE, and three after EFTR). All the patients improved after conservative treatments, such as the deprivation of food and water, rehydration, or rational intravenous use of antibiotics. Postoperative perforation occurred in one patient undergoing ESD, and the patient improved after surgery. The incidence of complications in different degrees of complete resection were not statistically significant $(P>$ 0.05). See Table 1 and Figure 2.

\section{Pathological and Immunohistochemical results}

Apart from the three patients who had partially excised specimens, the specimens of the 92 patients who underwent endoscopic treatment had complete surgical margins. The transverse diameter of the largest tumor was $3.2 \mathrm{~cm}$, while the transverse diameter of the smallest was $0.5 \mathrm{~cm}$; the average transverse diameter was $1.2 \pm 0.8 \mathrm{~cm}$. Transverse diameters of less than $2 \mathrm{~cm}$ were found in 70 patients, and transverse diameters of $2-5 \mathrm{~cm}$ were found in 22 patients.

A total of 88 patients had a mitotic index $<5 / 50 \mathrm{HPFs}$, and four patients had a mitotic index $<6-10 / 50$ HPFs.

Table I Comparison of Five Surgical Methods for Gastric Stromal Tumors

\begin{tabular}{|l|c|c|c|c|c|c|}
\hline Surgical & $\begin{array}{c}\text { Complete } \\
\text { Methods }\end{array}$ & $\begin{array}{c}\text { Hemorrhage During } \\
\text { Operation [n(\%)] }\end{array}$ & \multicolumn{2}{|c|}{$\begin{array}{c}\text { Perforation During } \\
\text { Operation [n(\%)] }\end{array}$} & \multicolumn{2}{|c|}{ Postoperative Complications [n(\%)] } \\
\cline { 3 - 6 } & (\%) & $\begin{array}{c}\text { Coagulation } \\
\text { Syndrome }\end{array}$ & Perforation & Bleeding \\
\hline EBL & $7 / 9(77.8)$ & $0(0)$ & $3(33.3)$ & $0(0)$ & $0(0)$ & $0(0)$ \\
ESD & $36 / 37(97.3)$ & $0(0)$ & $6(16.2)$ & $2(5.4)$ & $1(2.7)$ \\
ESE & $27 / 27(100)$ & $2(7.4)$ & $4(14.8)$ & $2(7.4)$ & $0(0)$ & $0(0)$ \\
EFTR & $15 / 15(100)$ & $0(0)$ & $-*$ & $3(20.0)$ & $0(0)$ & $0(0)$ \\
STER & $4 / 4(100)$ & $0(0)$ & $0(0)$ & $0(0)$ & $0(0)$ & $0(0)$ \\
\hline
\end{tabular}

Note: *Perforation in EFTR were not included.

Abbreviations: EBL, endoscopic band ligation; ESD, endoscopic submucosal dissection; ESE, endoscopic submucosal excavation; EFTR, endoscopic full-thickness resection; STER, submucosal tunneling endoscopic resection. 


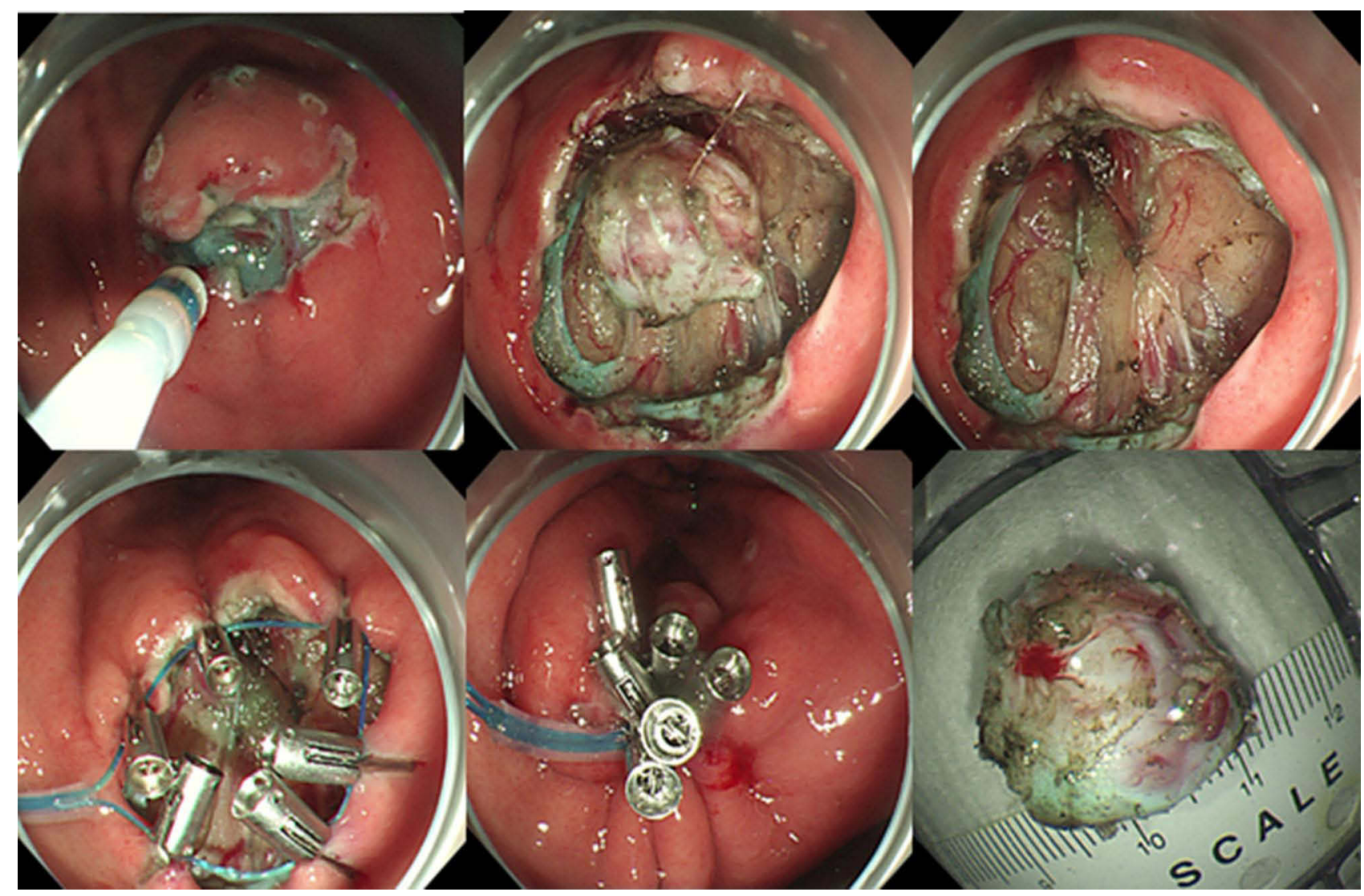

Figure 2 Complete endoscopic full-thickness resection of tumor; clips combined with purse-string suture closure of wounds.

A total of 82 patients tested positive for CD117, CD34, and DOG-1 in immunohistochemistry, among which three tested positive for SMA, 14 tested positive for vimentin, five tested positive for CD117, DOG-1, and vimentin, and five tested positive for $\mathrm{CD} 117$ and $\mathrm{CD} 34$ (including one patient who tested positive for SMA as well). According to the National Institutes of Health risk classification table (Table 2), ${ }^{5} 71$ patients had an extremely low risk, 16 had a low risk, three had a moderate risk, and two had a high risk. The pathological and immunohistochemical staining results are presented in Figures 3 and 4.

\section{EUS Image and Risk Classification}

In the pathological and immunohistochemical results, five cases were classified as being moderate or high risk. In order to avoid deviation in the final data analysis, we identified and analyzed the data of 16 patients who were pathologically confirmed to have GISTs after surgical resection but were not suitable for endoscopic resection after assessment by ordinary endoscopy and EUS. Based on the characteristics of the EUS images, including size (average diameter), echo type, the presence of anechoic fluid, and whether the echo was homogeneous, the patients were divided into four groups: extremely low risk, low risk, moderate risk, and high risk. Non-homogeneous internal echoes, anechoic fluid areas, and a hyperemic

Table 2 Modified NIH Classification System Proposed by Joensuu

\begin{tabular}{|c|c|c|c|}
\hline $\begin{array}{l}\text { Class of } \\
\text { Risk }\end{array}$ & $\begin{array}{c}\text { Tumor } \\
\text { Size }(\mathrm{cm})\end{array}$ & $\begin{array}{c}\text { Mitotic Index } \\
\text { (/50HPFs) }\end{array}$ & $\begin{array}{l}\text { Primary } \\
\text { Tumor } \\
\text { Location }\end{array}$ \\
\hline $\begin{array}{l}\text { Extremely } \\
\text { low risk }\end{array}$ & $<2.0$ & $<5$ & Any \\
\hline Low risk & $2.0-5.0$ & $<5$ & Any \\
\hline $\begin{array}{l}\text { Moderate } \\
\text { risk }\end{array}$ & $\begin{array}{c}<5.0 \\
5.1-10.0\end{array}$ & $\begin{array}{c}6-10 \\
<5\end{array}$ & $\begin{array}{l}\text { Gastric } \\
\text { Gastric }\end{array}$ \\
\hline High risk & $\begin{array}{c}\text { Any } \\
>10.0 \\
\text { Any } \\
>5.0 \\
\leq 5.0 \\
2.1-10.0\end{array}$ & $\begin{array}{l}\text { Any } \\
\text { Any } \\
>10 \\
>5 \\
>5 \\
\leq 5\end{array}$ & $\begin{array}{c}\text { Tumor rupture } \\
\text { Any } \\
\text { Any } \\
\text { Any } \\
\text { Non-gastric } \\
\text { Non-gastric }\end{array}$ \\
\hline
\end{tabular}

Abbreviations: NIH, National Institutes of Health; HPF, high-power field. 

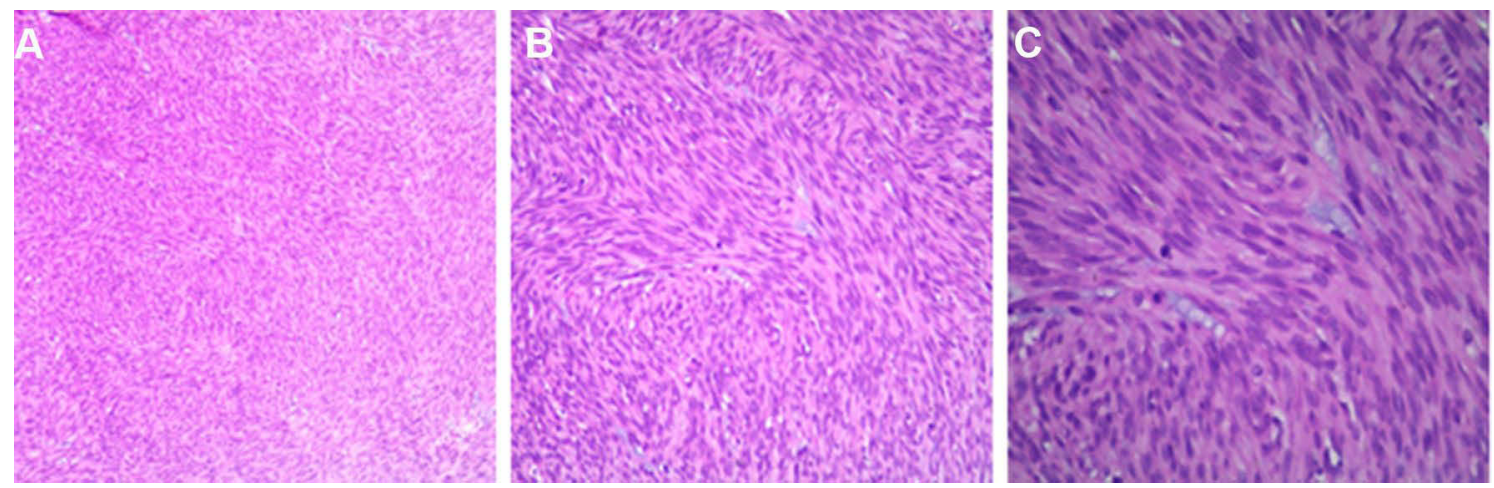

Figure 3 Electron microscope $(\times 40[$ A ] $\times 100$ [B], $\times 200$ [C]), hematoxylin and eosin staining of cross-sectioned gastrointestinal stromal tumor; spindle cells and mitotic figures can be seen.
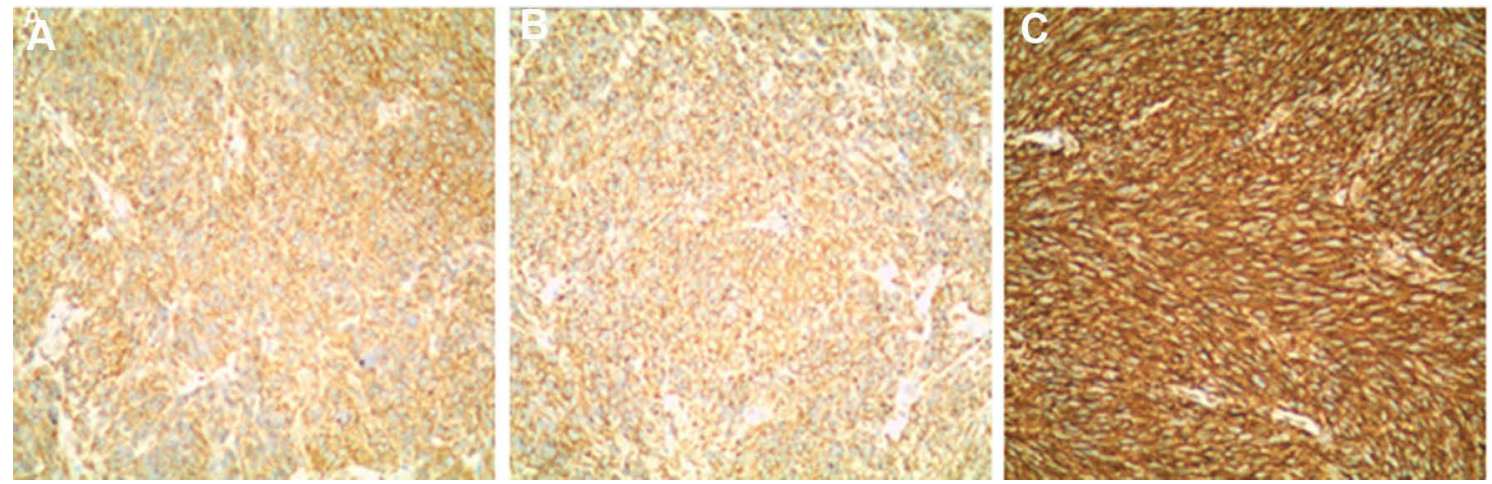

Figure 4 Immunohistochemical staining of gastrointestinal stromal tumor with DOGI (A), CDII7 (B), CD34 (C).

tumor surface accompanied by erosion were helpful in the identification of risk $(P<0.05)$. Tumor location and size were positively correlated with the risk of GISTs $(P<$ 0.05), but echo type, internal blood-flow signal, calcification, and surface characteristics (congestion, edema, or depression) of a tumor were not significantly correlated with the risk of GISTs $(P>0.05)$. See Table 3.

\section{Discussion}

At first, we collected cases in endanger the high-risk group of cases are relatively little, investigate its reason, may be in our normal endoscopy in patients with gastrointestinal stromal tumor and endoscopic ultrasonography examination, the part we will think that is not suitable for cases of endoscopic resection is recommended for surgical resection, and the diameter of the cases with tumors, internal echo and uneven surface hyperemia erosion characteristics of the total information complete 16 patients, so we get more complete and objective conclusion.

A previous study found that the invasiveness of a GIST increases with tumor diameter. ${ }^{6}$ Other studies have found that the diameter of a tumor mass larger than $3.0 \mathrm{~cm}$ and the presence of ulcers on the surface of a tumor suggest that a GIST has high malignant potential. ${ }^{6-12}$ These findings are consistent with the results of the present study.

Current EUS-based technologies, such as EUS-guided fine-needle aspiration biopsy, EUS-guided Trucut aspiration biopsy, and harmonic contrast-enhanced EUS (CH-EUS), have higher accuracy in the diagnosis of GISTs. ${ }^{13-15}$ A metaanalysis of four studies of 187 patients found that CH-EUS was valuable in the differential diagnosis of GISTs from submucosal lesions. ${ }^{16}$ Furthermore, a study conducted by Lee et $\mathrm{al}^{17}$ found that, for interstitial tumors with a diameter of $2-5 \mathrm{~cm}$, the EUS scoring system based on digital image analysis was helpful in predicting and diagnosing GIST.

This classification criteria recommended in the Asian Consensus Guidelines for the Diagnosis and Treatment of Gastrointestinal Stromal Tumors is also recommended for reference in the new edition of the Consensus of Experts on the Diagnosis and Expert Consensus on Endoscopic Diagnosis and Treatment of Gastrointestinal Stromal 
Table 3 Analysis of Ultrasound and General Endoscopic Features of Differentiated Gradient Stromal Tumors at Different Risks

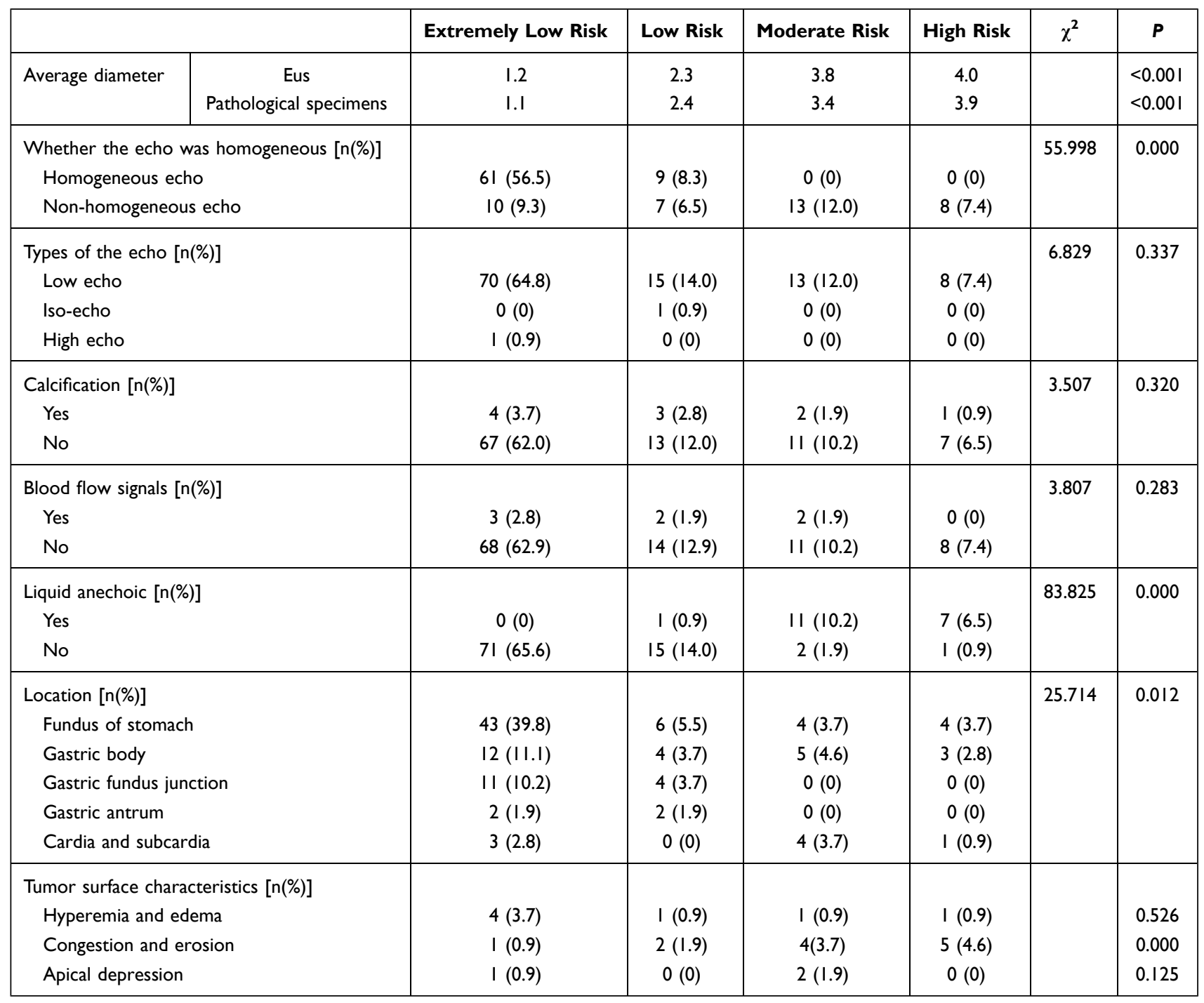

Abbreviation: EUS, endoscopic ultrasonography.

Tumors in China. ${ }^{4,18}$ The results of the present study revealed that the EUS-assisted endoscopic resection of GISTs is highly feasible and safe. EUS can guide the selection of appropriate endoscopic resection methods by evaluating tumor size, source level, and relationship with surrounding organs and blood vessels. For example, EBL and ESD can be selected for resection in cases where the luminal growth is dominant, the echo is uniform, the mucosal muscularis and muscularis propria are derived, and the layers are clear; for cases with complete origin in the muscularis propria and clear stratification of the serous layer, STER can be selected; and EFTR can be used for excision in cases with intraluminal and extraluminal growth and even in cases where ultrasonography indicates that the main extraluminal growth is closely related to the serous layer. Of course, the feasibility and safety of EUS is also closely related to the operator's experience.

The conclusions of the present study are consistent with the findings of previous studies: Sun et $\mathrm{al}^{19}$ found that EBL was safe and effective for the removal of GISTs, with a lower incidence of complications (3.4\%) and recur-

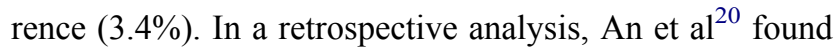
that EUS-assisted ESD was safe and feasible for the removal of GISTs. Other studies have revealed that EFTR and STER have excellent therapeutic effects in the removal of GISTs, and the therapeutic effect of both is the same. ${ }^{21,22}$ For tumors with a large diameter, uneven internal echo, anechoic fluid, and hyperemia and erosion on the tumor surface, surgical treatment can be selected as appropriate. However, there is still a lack of unified criteria to 
guide the treatment of GISTs based on EUS image characteristics and preoperative risk assessment.

The present study had some limitations. First, it was a retrospective study rather than a randomized controlled trial, and it was not blinded, so there was a risk of bias. Second, it was a single-center clinical trial with a small sample, so there was a risk of bias in choosing the research subjects.

\section{Conclusion}

The endoscopic resection of GISTs is feasible and safe. EUS is of great significance for the diagnosis and risk assessment of GISTs and can assist in the endoscopic resection of GISTs.

\section{Funding}

This study was supported by the international cooperation special project of Hebei Province Technology Department [No.18397738D].

\section{Disclosure}

The authors declare that they have no conflicts of interest.

\section{References}

1. von Mehren M, Joensuu H. Gastrointestinal stromal tumors. J Clin Oncol. 2018;36:136-143.

2. Søreide K, Sandvik OM, Søreide JA, Giljaca V, Jureckova A, Bulusu VR. Global epidemiology of gastrointestinal stromal tumours (GIST): a systematic review of population-based cohort studies. Cancer Epidemiol. 2016;40:39-46. doi:10.1016/j.canep.2015.10.031

3. Chou FF, Eng HL, Sheen-Chen SM. Smooth muscle tumors of the gastrointestinal tract: analysis of prognostic factors. Surgery. 1996;119:171-177. doi:10.1016/S0039-6060(96)80165-6

4. Digestive Endoscopic Tunnel Technique Cooperation Group of Chinese Medical Association Digestive Endoscopy Branch, Chinese Medical Doctor Association Endoscopic Physician Branch, Beijing Medical Association Digestive Endoscopy Branch. [Expert consensus on endoscopic diagnosis and treatment of gastrointestinal stromal tumors in China (2020, Beijing)]. Chin J Gastrointest Endosc. 2020;7:176-185. Chinese

5. Joensuu H. Risk stratification of patients diagnosed with gastrointestinal stromal tumor. Hum Pathol. 2008;39:1411-1419. doi:10.1016/j. humpath.2008.06.025

6. Joensuu H, Hohenberger P, Corless CL. Gastrointestinal stromal tumour. Lancet. 2013;382:973-983. doi:10.1016/S0140-6736(13) 60106-3

7. Harlan LC, Eisenstein J, Russell MC, Stevens JL, Cardona K. Gastrointestinal stromal tumors: treatment patterns of a population-based sample. $J$ Surg Oncol. 2015;111:702-707. doi: $10.1002 /$ jso. 23879

8. Jeon SW, Park YD, Chung YJ, et al. Gastrointestinal stromal tumors of the stomach: endosonographic differentiation in relation to histological risk. J Gastroenterol Hepatol. 2007;22:2069-2075. doi:10.1111/ j.1440-1746.2006.04767.x

9. Hayashi K, Kamimura K, Hosaka K, et al. Endoscopic ultrasound-guided fine-needle aspiration for diagnosing a rare extraluminal duodenal gastrointestinal tumor. World J Gastrointest Endosc. 2017;9:583-589. doi:10.4253/wjge.v9.i12.583
10. Hunt GC, Rader AE, Faigel DO. A comparison of EUS features between CD-117 positive GI stromal tumors and CD-117 negative GI spindle cell tumors. Gastrointest Endosc. 2003;57:469-474. doi: $10.1067 / \mathrm{mge} .2003 .146$

11. Kim GH, Kim KB, Lee SH, et al. Digital image analysis of endoscopic ultrasonography is helpful in diagnosing gastric mesenchymal tumors. BMC Gastroenterol. 2014;14:7. doi:10.1186/1471-230X-14-7

12. Peng CY, Lv Y, Xu GF. [Evaluation of preoperative endoscopic ultrasonography for diagnosis and invasive risk assessment of gastric gastrointestinal stromal tumors: a single-center retrospective study]. Chin J Digest Endosc. 2015;32:361-366. Chinese

13. Okasha HH, Naguib M, El Nady M, et al. Role of endoscopic ultrasound and endoscopic ultrasound guided fine-needle aspiration in endoscopic biopsy negative gastrointestinal lesions. Endosc Ultrasound. 2017;6:156-161. doi:10.4103/2303-9027.201086

14. Dumonceau JM, Polkowski M, Larghi A, et al. European Society of Gastrointestinal Endoscopy. Indications, results, and clinical impact of endoscopic ultrasound (EUS)-guided sampling in gastroenterology: European Society of Gastrointestinal Endoscopy (ESGE) Clinical Guideline. Endoscopy. 2011;43:897-912. doi:10.1055/s-0030-1256754

15. Kitano M, Sakamoto H, Komaki T, Kudo M. New techniques and future perspective of EUS for the differential diagnosis of pancreatic malignancies: contrast harmonic imaging. Dig Endosc. 2011;23 (Suppl 1):46-50. doi:10.1111/j.1443-1661.2011.01146.x

16. Tang JY, Tao KG, Zhang LY, et al. Value of contrast-enhanced harmonic endoscopic ultrasonography in differentiating between gastrointestinal stromal tumors: a meta-analysis. $J$ Dig Dis. 2019;20:127-134. doi:10.1111/1751-2980.12710

17. Lee MW, Kim GH, Kim KB, et al. Digital image analysis-based scoring system for endoscopic ultrasonography is useful in predicting gastrointestinal stromal tumors. Gastric Cancer. 2019;22:980-987.a. doi:10.1007/s10120-019-00928-w

18. Koo DH, Ryu MH, Kim KM, et al. Asian Consensus Guidelines for the Diagnosis and Management of Gastrointestinal Stromal Tumor. Cancer Res Treat. 2016;48:1155-1166. doi:10.4143/crt.2016.187

19. Sun S, Ge N, Wang C, Wang M, Lü Q. Endoscopic band ligation of small gastric stromal tumors and follow-up by endoscopic ultrasonography. Surg Endosc. 2007;21:574-578. doi:10.1007/ s00464-006-9028-4

20. An W, Sun PB, Gao J, et al. Endoscopic submucosal dissection for gastric gastrointestinal stromal tumors: a retrospective cohort study. Surg Endosc. 2017;31:4522-4531. doi:10.1007/s00464-017$5511-3$

21. Li B, Qi ZP, Zhou PH, et al. [Therapeutic value of endoscopic full-thickness resection for small gastrointestinal stromal tumor in the fundus of stomach]. Chin J Pract Surg. 2017;37:1281-1285. Chinese

22. Tan Y, Tang X, Guo T, et al. Comparison between submucosal tunneling endoscopic resection and endoscopic full-thickness resection for gastric stromal tumors originating from the muscularis propria layer. Surg Endosc. 2017;31:3376-3382. doi:10.1007/ s00464-016-5350-7

23. Du C, Chai N, Linghu E, et al. Clinical outcomes of endoscopic resection for the treatment of gastric gastrointestinal stromal tumors originating from the muscularis propria: a 7-year experience from a large tertiary center in China. Surg Endosc. 2021. doi:10.1007/ s00464-021-08443-9

24. Eom BW, Kim CG, Kook MC, et al. Feasibility of Non-Exposure Simple Suturing Endoscopic Full-Thickness Resection in Comparison with Laparoscopic Endoscopic Cooperative Surgery for Gastric Subepithelial Tumors: results of Two Independent Prospective Trials. Cancers. 2021;13:1858. doi:10.3390/cancers 13081858

25. Yang X, Wang H, Dong Q, et al. An artificial intelligence system for distinguishing between gastrointestinal stromal tumors and leiomyomas using endoscopic ultrasonography. Endoscopy. 2021. doi:10.1055/a-1476-8931 


\section{Publish your work in this journal}

The International Journal of General Medicine is an international, peer-reviewed open-access journal that focuses on general and internal medicine, pathogenesis, epidemiology, diagnosis, monitoring and treatment protocols. The journal is characterized by the rapid reporting of reviews, original research and clinical studies across all disease areas. The manuscript management system is completely online and includes a very quick and fair peer-review system, which is all easy to use. Visit http://www.dovepress.com/ testimonials.php to read real quotes from published authors.

Submit your manuscript here: https://www.dovepress.com/international-journal-of-general-medicine-journal 